\title{
Reduction in thermal conductivity of Bi-Te alloys through grain refinement method
}

\author{
SOMA DUTTA*, V SHUBHA and T G RAMESH \\ Materials Science Division, National Aerospace Laboratories, PO Box 1779, Kodihalli, Bangalore 560 017, India
}

MS received 10 August 2011; revised 11 April 2012

\begin{abstract}
Ternary alloys of thermoelectric materials Bi-Sb-Te and Bi-Se-Te of molecular formula, $\mathrm{Bi}_{0.5} \mathrm{Sb}_{1.5} \mathrm{Te}_{3}$ ( $p$ type) and $\mathrm{Bi}_{0.36} \mathrm{Se}_{0.064} \mathrm{Te}_{0.576}(n$ type), were prepared by mechanical alloying method. The preparation of materials by mechanical alloying method has effectively reduced the thermal conductivity by generating a large number of induced grain boundaries with required degree of disorder. The process of frequent milling was adapted for grain refinement. Substantial reduction in thermal conductivity was achieved due to nano-structuring of these alloys. Thermal conductivity values were found to be very low at room temperature, $0.5 \mathrm{~W} / \mathrm{mK}$ and $0.8 \mathrm{~W} / \mathrm{mK}$, respectively for $p$ and $n$ type materials.
\end{abstract}

Keywords. Bismuth telluride; mechanical alloying; grain boundary; thermal conductivity.

\section{Introduction}

Bismuth telluride-based materials are widely used for thermoelectric devices around room temperature. Conventional melting techniques were used for preparation of these alloys leading to fabrication of thermoelectric devices. The unidirectional grown materials are inclined to cleavage fracture due to their weak Van der Waals bonding between the hexagonally stacked five-atomic layers, $\mathrm{Te}-\mathrm{Bi}-\mathrm{Te}-\mathrm{Bi}-\mathrm{Te} . \mathrm{Bi}_{2} \mathrm{Te}_{3}-$ based single crystals are easily fractured during the cutting process or operation of the modules. Getting the nanostructure into bulk materials remains a key challenge to improve the mechanical strength and electro-thermal properties. Powder metallurgical methods offer higher strength in these materials due to nano-size grains improving electro-thermal properties.

Mechanical alloying (MA) method proved to be a versatile alternative to other processing routes in preparing nanostructured materials with a broad range of chemical compositions (Rowe et al 1981; Hyun et al 1998; Martin-Lopez et al 1999; Yang et al 2000, 2001, 2006a, b; Suryanarayana 2001). Although there are numerous techniques to produce nanostructured materials, this method has become more popular due to its simplicity, capacity for large-scale production and cost effectiveness. MA process is greatly affected by number of factors that play very important role in the preparation of homogeneous materials. The properties of the milled powders (particle size distribution, degree of disorder or amorphization and final stoichiometry) depend on the milling conditions. MA is a process of alloy formation in the solid phase by means of repeated mechanical impact (Bouad et al 2000). The method is suitable even for those alloys

\footnotetext{
*Author for correspondence (som@nal.res.in)
}

which are difficult to obtain via solidification processes due to their unfavourable phase diagram.

Nanostructuring approach has proven to be very efficient to reduce the lattice contribution to the thermal conductivity in many thermoelectric material systems (Poudel et al 2008; Lan et al 2010; Yan et al 2011; Wang et al 2011) thereby improving the figure-of-merit of thermoelectric material (Rowe et al 1981).

Present investigation on thermal conductivity of $p$ and $n$ type $\mathrm{Bi}-\mathrm{Te}$ alloys primarily deals with the aim of reducing thermal conductivity without having any adverse effect on the electrical power factor $S^{2} \sigma$, where $S$ is the thermoelectric power and $\sigma$ the electrical conductivity. Lowering thermal conductivity without compromising on the power factor $S^{2} \sigma$ is a challenge in these $\mathrm{Bi}-\mathrm{Te}-\mathrm{Sb}-\mathrm{Se}$ alloys. Using grain refinement method, higher thermopower $(S) \sim 216 \mu \mathrm{V} /{ }^{\circ} \mathrm{C}$ and electrical conductivity, $1.02 \times 10^{5} \mathrm{ohm}^{-1} \mathrm{~m}^{-1}$ were achieved for $p$-type material $\left(\mathrm{Bi}_{0.5} \mathrm{Sb}_{1.5} \mathrm{Te}_{3}\right)$. These values compare favourably with that reported in the literature (Yang et al 2001). However, thermopower value of $-170 \mu \mathrm{V} /{ }^{\circ} \mathrm{C}$ was obtained for $n$-type $\mathrm{Bi}_{0.36} \mathrm{Se}_{0.064} \mathrm{Te}_{0.576}$ and electrical conductivity of $0.85 \times 10^{5} \mathrm{ohm}^{-1} \mathrm{~m}^{-1}$ which are in agreement with the literature values (Yang et al 2006a, b). In this paper, we report preparation and experimental results on characterization of these materials, highlighting the feature of low thermal conductivity in correlation with the fine grain structures.

\section{Experimental}

\subsection{Preparation}

$\mathrm{Bi}_{2} \mathrm{Te}_{3}$ with stoichiometric compositions of $\mathrm{Bi}_{0.5} \mathrm{Sb}_{1.5} \mathrm{Te}_{3}$ (with $4 \mathrm{wt} \%$ extra Te) and $\mathrm{Bi}_{0.36} \mathrm{Se}_{0.064} \mathrm{Te}_{0.576}$ were prepared 
by high-energy ball milling using planetary ball mill Fritsch Pulverisette 6. The elemental granules of bismuth (Bi), tellurium (Te), antimony ( $\mathrm{Sb}$ ) and selenium (Se) with $4 \mathrm{~N}$ purity were crushed down to pieces smaller than $10 \mathrm{~mm}$. The starting materials were weighed according to the composition and then subjected to mechanical alloying in planetary ball mill under purified argon atmosphere. For milling, hardened tungsten carbide vessel was used with tungsten carbide grinding balls of $30 \mathrm{~mm}$ diameter. The weight ratio of ball to powder was maintained at 10:1. The frequency of the system was optimized at $400 \mathrm{rpm}$ for the preparation of $\mathrm{Bi}_{0.5} \mathrm{Sb}_{1.5} \mathrm{Te}_{3}$ and $450 \mathrm{rpm}$ for $\mathrm{Bi}_{0.36} \mathrm{Se}_{0.064} \mathrm{Te}_{0.576}$ samples based on the impact energy. The pulverization force (impact energy), $F$, generated by the ball mill was calculated using the formula given below:

$$
F=n\left(\frac{m}{d}\right) v^{2} \frac{t}{w}
$$

where $n$ is the number of balls, $m$ the mass of the balls, $d$ the pot diameter, $v$ the ball speed, $t$ the mixing time and $w$ the loaded mass. The ball speed can be expressed as $v=\left(d^{\prime} \pi n^{\prime} / 60\right), d^{\prime}$ is the mill diameter and $n^{\prime}$ the rotation speed of the mill. Milling conditions were optimized based on the generated pulverization force. It was calculated that a force of $3 \mathrm{kN}$ would be generated when the frequency of the mill is set at $400 \mathrm{rpm}$ and $5 \mathrm{kN}$ at $450 \mathrm{rpm}$. Since the melting point of $p$-type material is lower than that of $n$-type material, $3 \mathrm{kN}$ force was sufficient to prepare single phase $p$-type material and $5 \mathrm{kN}$ for the $n$-type material which is equivalent to the heat energy required for melting these materials. For optimizing the time of milling, repeated X-ray analysis was done till minimum particle size was achieved which did not change with further process of milling depending on the limit of grindability of the material (Pilchak et al 2007).

Initially the particle size decreased with milling time according to the following relationship:

$$
d(t)=\left(d_{0}-d_{\mathrm{L}}\right) \exp \left(\frac{-t}{\tau}\right)+d_{\mathrm{L}},
$$

where $d_{0}$ is the initial powder particle size (at an elapsed milling time of zero), $\tau$ a time constant and $d_{\mathrm{L}}$ the grindability limit. Thus $d_{0}-d_{\mathrm{L}}$ represents the total change from the initial particle size (milling time, $t=0$ ) to the grindability limit, $d_{\mathrm{L}}$, for which particle size does not change as the milling time increases. The effect of milling on the particle size was observed for a milling time of $10 \mathrm{~h}$ with ball-to-powder weight ratio of 10:1 where the mean particle size achieved was $12 \mathrm{~nm}$ for $p$-type and $14 \mathrm{~nm}$ for $n$-type.

X-ray diffraction pattern (XRD) of the milled powder confirmed the alloy formation. Powder was sieved for separating the fine grain (particle size, $<100 \mathrm{~nm}$ ) and was repeatedly milled for $2 \mathrm{~h}$ to get homogenized material. XRD was repeated for this fine grain milled powder to reconfirm the phase. The nano-size powder was compacted under a uniaxial pressure of $50 \mathrm{MPa}$ followed by annealing at a temperature close to the melting point of these materials. Metallurgically it is well known that annealing of metallic alloys close to its melting point normally aligns all the atoms into a minimum energy state which produces toughness and hard structures. It also facilitates grain growth and reduces the porosity. The final XRD was taken on the annealed sample to compare the shift in peak position and change in intensity compared to that of the milled powder, if any. Density of the samples was measured by the Archimedes method and was found to be closer to the value calculated using mass and volume of the sample.

\subsection{Characterization}

X-ray diffraction data was used to calculate crystallite/ particle size from the Fourier line shape analysis following the Warren and Averbach (1950) and Balzar and Ledbetter (1993) methods. Lattice parameters were determined using least-squares refinement method with the help of a computer package, Powd Mult, developed by E Wu, School of Physical Science, Flinders University of South Australia. Grain structure, grain distribution and compositional analysis of the powder were performed by scanning electron microscopy (SEM). The thermal conductivity was measured using laser flash technique at different temperatures ranging from room temperature to $300{ }^{\circ} \mathrm{C}$.

\section{Results and discussion}

Figure 1(a) and (b) gives XRD patterns of annealed compacts of $\mathrm{Bi}_{0.5} \mathrm{Sb}_{1.5} \mathrm{Te}_{3}$ and $\mathrm{Bi}_{0.36} \mathrm{Se}_{0.064} \mathrm{Te}_{0.576}$ alloys. All the reflection peaks were indexed for hexagonal system (JCPDS 15-0863). The least square refined lattice parameters, unit cell volume, crystallite size and rms strain of these materials are given in table 1 . The calculated volume weighted distributions $(p V)$ and crystallite size as a function of the Fourier length $(L)$ for $\mathrm{Bi}_{0.5} \mathrm{Sb}_{1.5} \mathrm{Te}_{3}$ and $\mathrm{Bi}_{0.36} \mathrm{Se}_{0.064} \mathrm{Te}_{0.576}$ samples are given in figure 2 (a) and (b), respectively. The average (60\% in volume) crystallite (particle) size estimated from X-ray data was $12 \mathrm{~nm}$ for $\mathrm{Bi}_{0.5} \mathrm{Sb}_{1.5} \mathrm{Te}_{3}$ and $14 \mathrm{~nm}$ for $\mathrm{Bi}_{0.36} \mathrm{Se}_{0.064} \mathrm{Te}_{0.576}$.

Figure 3(a) and (b) show high resolution micrographs of fractured surfaces of annealed $\mathrm{Bi}_{0.5} \mathrm{Sb}_{1.5} \mathrm{Te}_{3}$ and $\mathrm{Bi}_{0.36} \mathrm{Se}_{0.064} \mathrm{Te}_{0.576}$ samples. It was observed that for both $p$ and $n$ type materials, the average grain size was below $50 \mathrm{~nm}$. Nano-size and phase homogenization through grain refinement process improves the thermoelectric efficiency by decreasing the lattice thermal conductivity. The effect of nanostructure is reflected in thermal properties of these materials which will be discussed at a later stage.

The measured density of annealed $p$ and $n$ type materials were 6.64 and $7.3 \mathrm{~g} / \mathrm{cm}^{3}$, respectively which was about $96 \%$ of the theoretical density $\left(6.878 \mathrm{~g} / \mathrm{cm}^{3}\right.$ for $p$-type and $7.7 \mathrm{~g} / \mathrm{cm}^{3}$ for $n$-type). 
Figure 4 represents thermal conductivity data of $\mathrm{Bi}_{0.5} \mathrm{Sb}_{1.5} \mathrm{Te}_{3}$ and $\mathrm{Bi}_{0.36} \mathrm{Se}_{0.064} \mathrm{Te}_{0.576}$ materials at different temperatures. The main observations of this plot are: (i) low thermal conductivity values of these materials at room temperature $\left(0.5 \mathrm{~W} / \mathrm{mK}\right.$ for $\mathrm{Bi}_{0.5} \mathrm{Sb}_{1.5} \mathrm{Te}_{3}$ and $0.8 \mathrm{~W} / \mathrm{mK}$ for $\left.\mathrm{Bi}_{0.36} \mathrm{Se}_{0.064} \mathrm{Te}_{0.576}\right)$ compared to that of the reported one (Poudel et al 2008; Mehta et al 2012; Soni et al 2012) and (ii) increase in thermal conductivity values with temperature.
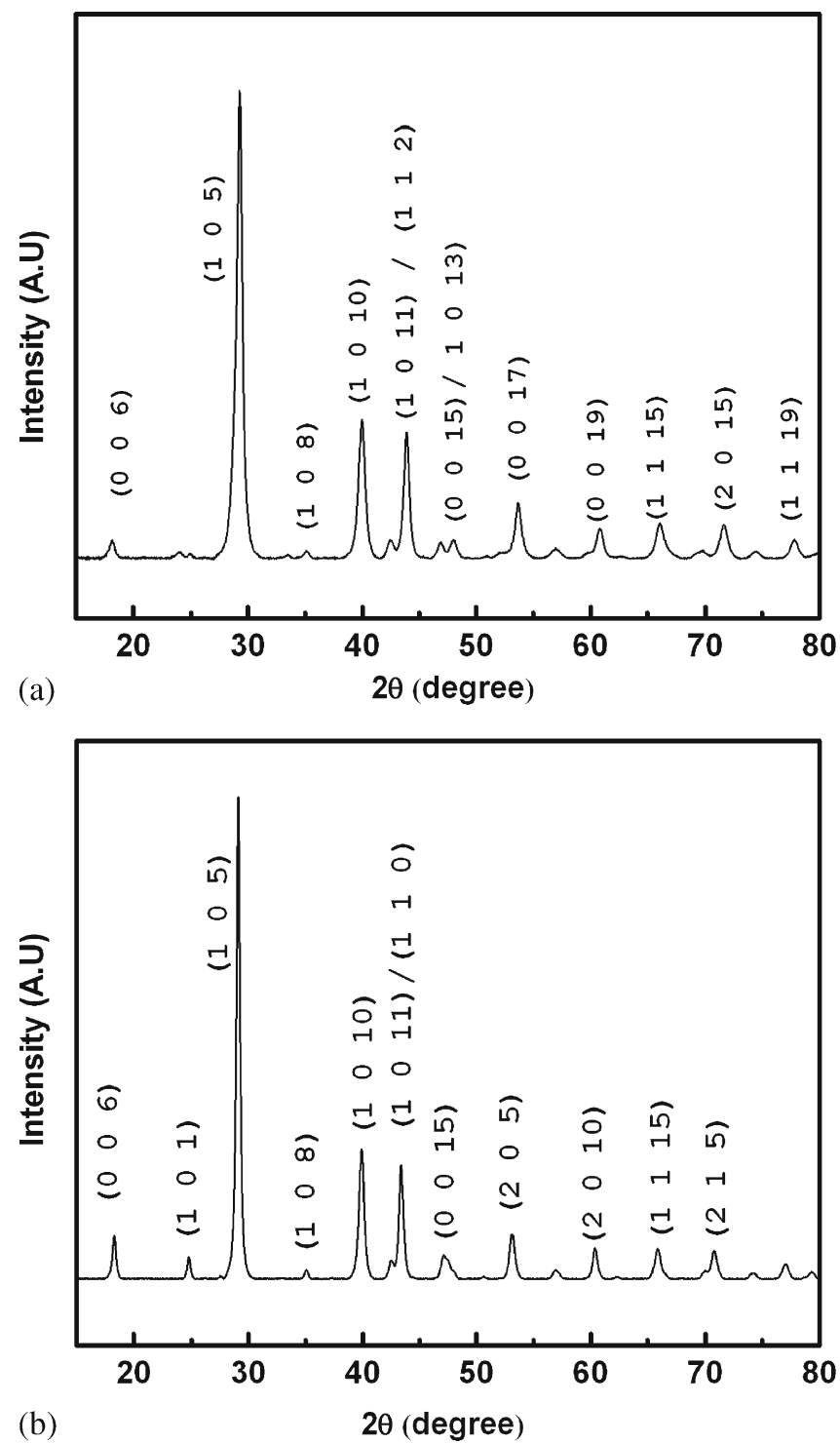

Figure 1. XRD pattern of (a) $\mathrm{Bi}_{0.5} \mathrm{Sb}_{1.5} \mathrm{Te}_{3}$ and (b) $\mathrm{Bi}_{0.36} \mathrm{Se}_{0.064} \mathrm{Te}_{0.576}$ alloys.
(Thermal conductivity value reaches close to $1.5 \mathrm{~W} / \mathrm{mK}$ for both $p$ and $n$ type materials at $300{ }^{\circ} \mathrm{C}$.)

The thermal conductivity $(\kappa)$ of a semiconductor has two main components: the lattice (phonon) component $\left(\kappa_{1}\right)$ and the electronic (or hole) component $\left(\kappa_{\mathrm{el}}\right)$, i.e. $\kappa=\kappa_{\mathrm{el}}+\kappa_{1}$ (Goldsmid 1995). The fact that a significant $\kappa_{\mathrm{el}}$ is invariably present needs to be taken into account in any thermal conductivity minimization program. The electronic thermal conductivity for semiconductors whose carriers are all of one sign can be written according to the Wiedemann-Franz law:

$$
\kappa_{\mathrm{el}}=A\left(\frac{K}{e}\right)^{2} \sigma T,
$$
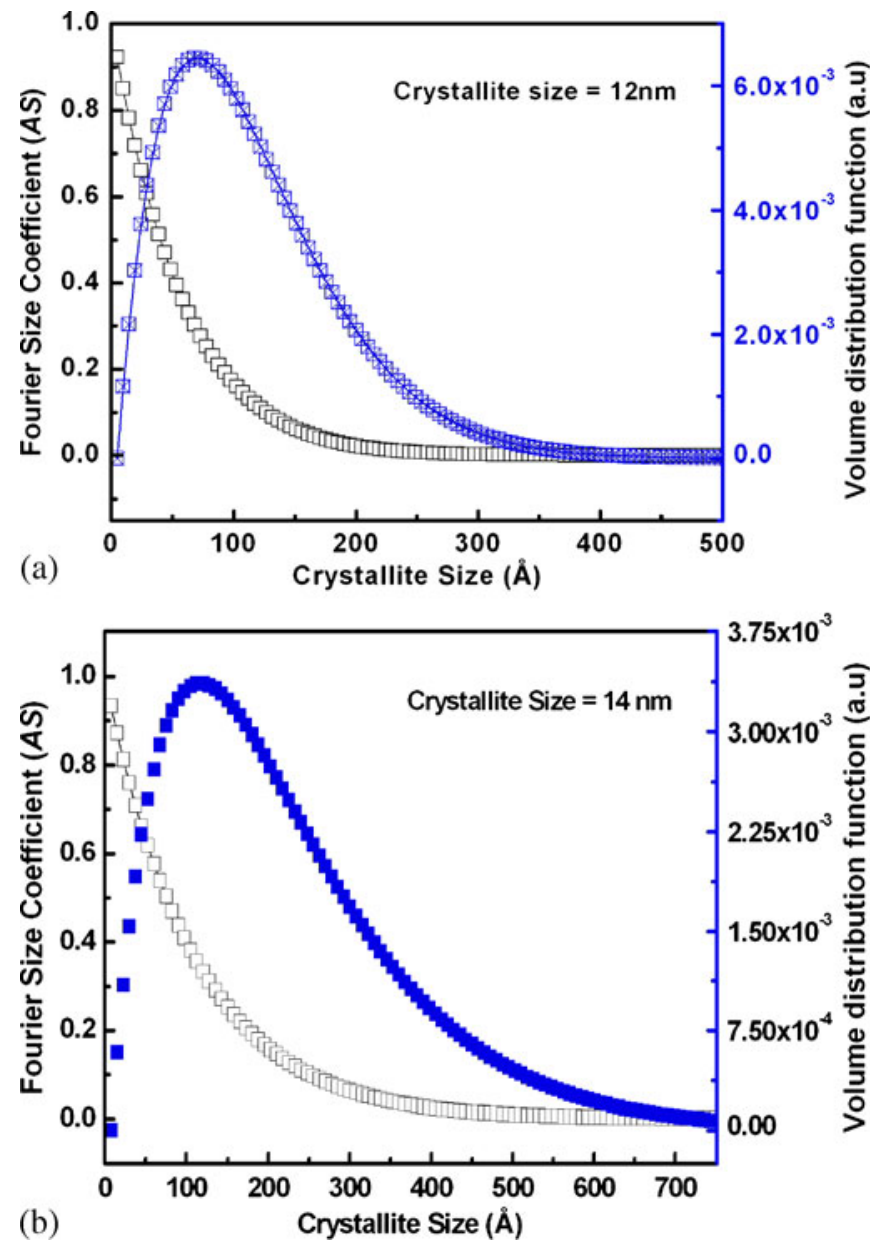

(b)

(b) Figure 2. Fourier line profile analysis plots for (a) $\mathrm{Bi}_{0.5} \mathrm{Sb}_{1.5} \mathrm{Te}_{3}$ and (b) $\mathrm{Bi}_{0.36} \mathrm{Se}_{0.064} \mathrm{Te}_{0.576}$ samples.

Table 1. Lattice parameters, unit cell volume, crystallite size and rms strain of (a) $\mathrm{Bi}_{0.5} \mathrm{Sb}_{1.5} \mathrm{Te}_{3}$ and (b) $\mathrm{Bi}_{0.36} \mathrm{Se}_{0.064} \mathrm{Te}_{0.576}$ alloys.

\begin{tabular}{lccccc}
\hline Material & $a(\AA)$ & $c(\AA)$ & Volume $\left(\AA^{3}\right)$ & Size $(\mathrm{nm})$ & Strain $\left\langle e^{2}\right\rangle^{1 / 2}$ \\
\hline (a) $\mathrm{Bi}_{0.5} \mathrm{Sb}_{1.5} \mathrm{Te}_{3}$ & 4.1637 & 28.9716 & 434.97 & 12 & $0.63 \times 10^{-2}$ \\
(b) $\mathrm{Bi}_{0.36} \mathrm{Se}_{0.064} \mathrm{Te}_{0.576}$ & 4.1733 & 28.9512 & 436.68 & 14 & $0.42 \times 10^{-2}$ \\
\hline
\end{tabular}



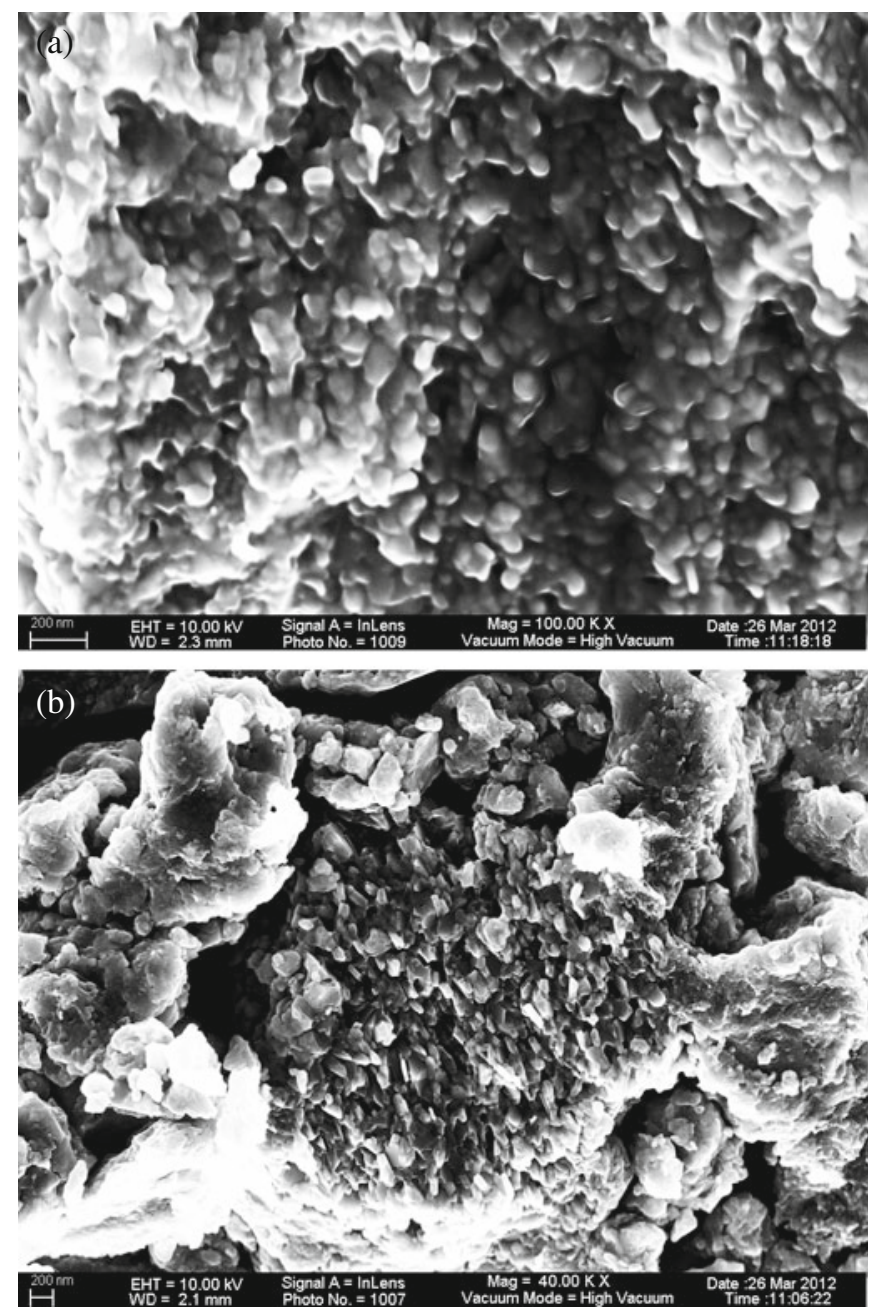

Figure 3. Fractographs of (a) $\mathrm{Bi}_{0.5} \mathrm{Sb}_{1.5} \mathrm{Te}_{3}$ and (b) $\mathrm{Bi}_{0.36} \mathrm{Se}_{0.064}$ $\mathrm{Te}_{0.576}$ alloys.

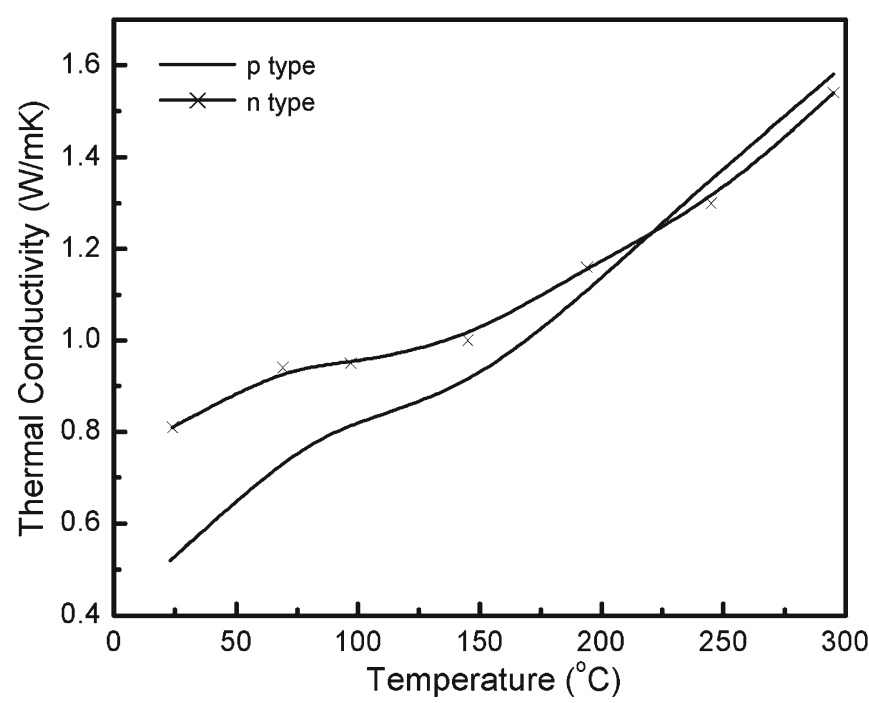

Figure 4. Plot of thermal conductivity vs temperature of $\mathrm{Bi}_{0.5} \mathrm{Sb}_{1.5} \mathrm{Te}_{3}$ ( $p$ type) and $\mathrm{Bi}_{0.36} \mathrm{Se}_{0.064} \mathrm{Te}_{0.576}(n$ type) materials. where $K$ is the Boltzmann constant, $e$ the electronic charge, $\sigma$ the electrical conductivity and $T$ the absolute temperature. $A$ is proportionality constant, equal to 2 in a non-degenerate electron system, if the mean free path, $l$ is independent of the energy $\varepsilon$; in general $A=r+2$, where $r$ is the exponent in the law $l \infty \varepsilon^{\mathrm{r}}$. For a material with particular carrier concentration, the parameter $K / e$ is constant at a given temperature $T$. Then $\kappa_{\mathrm{el}}$ is directly proportional to electrical conductivity $(\sigma)$. Thus, it is impossible to reduce the electronic contribution to thermal conductivity without lowering the electrical conductivity. The electrical conductivity of these materials measured at room temperature was $\sim 1.02 \times$ $10^{5} \mathrm{ohm}^{-1} \mathrm{~m}^{-1}$ for $\mathrm{Bi}_{0.5} \mathrm{Sb}_{1.5} \mathrm{Te}_{3}$ and $0.85 \times 10^{5} \mathrm{ohm}^{-1} \mathrm{~m}^{-1}$ for $\mathrm{Bi}_{0.36} \mathrm{Se}_{0.064} \mathrm{Te}_{0.576}$, which agrees well with the reported value (Goldsmid 1995). The reason for low thermal conductivity of these materials is due to the reduction in lattice contribution to thermal conductivity and not from that of electronic part. In general, $\kappa_{1}$ for polycrystalline alloy is smaller than that of the single crystal due to the fact that $\kappa_{1}$ is inversely proportional to the mass of the unit cell (Rowe et al 1981) and grain size (Schilz et al 1999) which facilitates the phonon scattering at the grain boundaries resulting in lowering of thermal conductivity of the material. Any mechanism that scatters phonons more effectively than the electron (or holes) is likely to enhance the electrical to thermal conductivity ratio. Among the important scattering mechanisms that tend to reduce the phonon mean free path are scattering by other phonons, lattice defects, electrons holes and grain boundaries. A very large number of grain boundaries are generated when mechanical alloying is employed to prepare nano-size powder of these materials followed by repetitive grain refinement method. The fine grain structure of these materials with large grain boundaries facilitates grain boundary scattering which effectively reduces the lattice thermal conductivity. Grain boundary scattering is the dominant scattering mechanism in samples with smaller grain sizes (Zhao et al 2011). The other factors which play important role in thermal conductivity reduction are phonon frequency and phonon mean free path. The phonon mean free path $(\lambda)$ is limited by crystal dimension. Fine grain boundaries effectively scatter short wavelength phonons with the net heat conduction due to long wavelength phonons. This process of phonon scattering by grain boundaries helps in decreasing the thermal conductivity of these materials. Studies on lead telluride based alloys reveal that a grain size of $1 \mu \mathrm{m}$ could reduce the lattice thermal conductivity of these alloys by 11-13\% (Bhandari and Rowe 1983; Rowe and Bhandari 1985). Nanostructured $p$-type BiSbTe bulk alloy achieved $83 \%$ thermal conductivity reduction compared with its ingot counterpart at $250{ }^{\circ} \mathrm{C}$ (Poudel et al 2008).

The effect of temperature on thermal conductivity (figure 4) will be discussed in this section. A marked increase in thermal conductivity from 0.8 to $1.4 \mathrm{~W} / \mathrm{mK}$ ( $\sim 75 \%$ ) was observed at high temperatures $\left(300{ }^{\circ} \mathrm{C}\right)$. Generally, the thermal conductivity increases if carriers of both signs are present. The diffusion of both minor and major charge carriers (electron and hole) depending on $n$ or $p$ type 
materials, transfers higher thermal energy compared to the single carriers (electron or hole). At higher temperatures, the contribution to the thermal conductivity from minority carriers dominates over that of the majority carriers, resulting in thermal conductivity increase. Increase in thermal conductivity with temperature (figure 4) proves that both the carriers take part in thermal conduction.

Thermal conductivity due to participation of both the carriers can be written mathematically as:

$$
k_{\text {pair }}=\frac{K^{2}}{e^{2}} T \frac{\sigma_{1} \sigma_{2}}{\sigma_{1}+\sigma_{2}}\left(\frac{\Delta E_{0}}{2 K T}+2+r\right)^{2},
$$

where $\sigma_{1}$ and $\sigma_{2}$ are the electrical conductivities assigned respectively to the holes and electrons.

Equation (2) can be written as

$$
k_{\text {pair }}=L\left(\sigma_{1}+\sigma_{2}\right)+L \frac{2 \sigma_{1} \sigma_{2}}{\sigma_{1}+\sigma_{2}}\left(\frac{\Delta E_{0}}{2 K T}+2+r\right)^{2},
$$

where $L=A(K / e)^{2}$ is the coefficient of $\sigma$ in (1), in which $A=(2+r)$.

The first term in (3) gives the Wiedemann-Franz law (1), whereas the second term gives extra thermal conductivity due to diffusion of electron and hole pairs. It is important to note that as a function of temperature, electrical conduction is primarily by carriers of one sign (majority carriers), whilst carriers of other sign (minority carriers) participate in the current transfer to a smaller extent. The effect of the minority carriers on thermal conductivity is appreciable (Ioffe 1960) as a function of temperature. When the minority carrier current amounts to only $10 \%$ of the majority carrier current, then $\left(\sigma_{1} \sigma_{2} /\left(\sigma_{1}+\sigma_{2}\right)\right)=0 \cdot 1\left(\sigma_{1}+\sigma_{2}\right)$ and thermal conductivity due to diffusion of both the carriers $\left(k_{\text {pair }}\right)$ turns out to be 8 times that of single carrier.

In bismuth telluride $\left(\mathrm{Bi}_{2} \mathrm{Te}_{3}\right)$ with a forbidden gap width of $0.16 \mathrm{eV}$, intrinsic conduction has substantial effect on the thermal conductivity even below room temperature. Thus, above room temperature, the additional conductivity due to diffusion of the pairs becomes remarkable, although the concentration of minority carriers is very less compared to that of the majority carriers. The appreciable increase of thermal conductivity as it is seen from figure 4 is due to the participation of both types of carriers.

\section{Conclusions}

In this paper, preparation of nano-size $\mathrm{Bi}_{0.5} \mathrm{Sb}_{1.5} \mathrm{Te}_{3}$ ( $p$ type $\mathrm{Bi}_{2} \mathrm{Te}_{3}$ ) and $\mathrm{Bi}_{0.36} \mathrm{Se}_{0.064} \mathrm{Te}_{0.576}$ ( $n$ type $\mathrm{Bi}_{2} \mathrm{Te}_{3}$ ) materials by employing mechanical alloying method is presented. With the optimized process control parameters, fine-grained material with particle size of $12-14 \mathrm{~nm}$ was obtained for $p$ and $n$ types, respectively. A profound effect of microstructure refinement resulted in thermal conductivity reduction. Experimental observations revealed the fact that the preparation of fine-grained material could effectively reduce thermal conductivity. Reduction in thermal conductivity is attributed to lattice phonon scattering. The increase in thermal conductivity with temperature is explained due to preferential contribution from minority charge carriers.

\section{References}

Balzar D and Ledbetter H 1993 J. Appl. Crystallogr. 2697

Bhandari C M and Rowe D M 1983 J. Phys. D: Appl. Phys. L75 16

Bouad N, Marin-Ayral R M and Tedenac J C 2000 J. Alloys Compd. 297312

Goldsmid H J 1995 Handbook of thermoelectrics (ed.) D M Rowe (Boca Raton, Florida: CRC Press) Ch. 3

Hyun D B, Oh T S and Hwang C W 1998 J. Mater. Sci. 335598

Ioffe A F 1960 Physics of semiconductors (London: Infosearch Limited)

Lan Y C, Minnich A J, Chen G and Ren Z F 2010 Adv. Funct. Mater. 20357

Martin-Lopez R, Dauscher A and Scherrer H 1999 Appl. Phys. A 68 597

Mehta R J, Zhang Y, Karthik C, Singh B, Siegel R W, Tasciuc T B and Ramanath G 2012 Nat. Mater. 11233

Pilchak A L et al 2007 Philos. Mag. 874567

Poudel B et al 2008 Science $\mathbf{3 2 0} 634$

Rowe D M, Shukla V S and Savvides N 1981 Nature 290765

Rowe D M and Bhandari C M 1985 Appl. Phys. Lett. 47256

Schilz J, Riffel M, Pixius K and Meyer H J 1999 Powder Technol. 105149

Soni A, Yanyuan Z, Ligen Y, Khiam A M K, Dresselhaus M S and Xiong Q 2012 Nano Lett. 121203

Suryanarayana C 2001 Prog. Mater. Sci. 461

Wang Z, Alaniz J E, Jang W, Garay J E and Dames C 2011 Nano Lett. 112206

Warren B E and Averbach B L 1950 J. Appl. Phys. 21595

Yan X et al 2011 Nano Lett. 11556

Yang J Y, Aizawa T, Yamamoto A and Ohta T 2000 J. Alloys Compd. 312326

Yang J Y, Aizawa T, Yamamoto A and Ohta T 2001 Mater. Chem. Phys. 7090

Yang J Y, Chen R G, Fan X A, Bao S Q and Zhu W 2006a J. Alloys Compd. 407330

Yang J Y, Fan X A, Chen R G, Zhu W, Bao S Q and Duan X K 2006b J. Alloys Compd. 41270

Zhao H et al 2011 Appl. Phys. Letts 99163101 